\title{
Cubic-spline interpolation to estimate effects of inbreeding on milk yield in first lactation Holstein cows
}

\author{
Makram J. Geha ${ }^{1}$, Jeffrey F. Keown ${ }^{1}$ and L. Dale Van Vleck ${ }^{1,2}$ \\ ${ }^{1}$ Department of Animal Science, University of Nebraska, Lincoln, NE, USA. \\ ${ }^{2}$ Roman L. Hruska U.S. Meat Animal Research Center, ARS, USDA, Lincoln, NE, USA.
}

\begin{abstract}
Milk yield records (305d, 2X, actual milk yield) of 123,639 registered first lactation Holstein cows were used to compare linear regression $\left(y=\beta_{0}+\beta_{1} X+e\right)$, quadratic regression, $\left(y=\beta_{0}+\beta_{1} X+\beta_{2} X^{2}+e\right)$ cubic regression $\left(y=\beta_{0}+\beta_{1} X+\right.$ $\left.\beta_{2} X^{2}+\beta_{3} X^{\beta}+e\right)$ and fixed factor models, with cubic-spline interpolation models, for estimating the effects of inbreeding on milk yield. Ten animal models, all with herd-year-season of calving as fixed effect, were compared using the Akaike corrected-Information Criterion (AICc). The cubic-spline interpolation model with seven knots had the lowest $\mathrm{AICc}$, whereas for all those labeled as "traditional", AICc was higher than the best model. Results from fitting inbreeding using a cubic-spline with seven knots were compared to results from fitting inbreeding as a linear covariate or as a fixed factor with seven levels. Estimates of inbreeding effects were not significantly different between the cubic-spline model and the fixed factor model, but were significantly different from the linear regression model. Milk yield decreased significantly at inbreeding levels greater than $9 \%$. Variance component estimates were similar for the three models. Ranking of the top 100 sires with daughter records remained unaffected by the model used.
\end{abstract}

Key words: Akaike's information criterion, cubic-spline interpolation, inbreeding, milk yield.

Received: April 23, 2010; Accepted: February 28, 2011.

\section{Introduction}

Commercial adoption of artificial insemination by the dairy industry has facilitated the intensification of selection for traits such as milk, fat and protein yields. Selection is largely based on Predicted Transmitting Ability (PTA), resulting from best linear unbiased prediction (BLUP) and selection indices. Both the moderate heritability of production traits and the use of BLUP have lead to the predominant use of specific family-lines of bulls (Weigel, 2001), especially in the Holstein population, which in turn has resulted in mating of related animals. A consequence of such matings is an increase in average inbreeding. The development of multiple ovulation and embryo transfer technology have also contributed to increasing levels of inbreeding by intensifying selection for high producing cows used as dams of AI bulls (Kearney et al., 2004). The average inbreeding coefficient in the Holstein population in the United States, as reported by the Animal Improvement Program Laboratory for animals born between January and June of 2009 (using 1960 as base year), was 5.50\% (ARS-AIPL, 2009). Several studies have shown the detrimental effects of inbreeding on several production, reproduction and health traits, even at low levels. For example, Hudson and Van Vleck (1984a,b) studied ef-

Send correspondence to Makram J. Geha. Department of Animal Science, University of Nebraska, Lincoln, NE 68583-0908, USA. E-mail: mjgeha@ huskers.unl.edu. fects of inbreeding on first lactation milk and fat production in five dairy breeds, using a linear regression approach. They reported decreased milk yield in inbred animals in all the five breeds, with an estimated loss of $21 \mathrm{~kg}$ of milk per $1 \%$ increase in the inbreeding coefficient in Holsteins. They also noted that the effects of inbreeding were non-linear, when inbreeding was modelled as a classification variable. With similar statistical models, Miglior et al. (1992) reported a significant reduction in total milk yield of $10 \mathrm{~kg}$ for each $1 \%$ increase in inbreeding in Canadian Jersey cattle. The Canadian group also reported the non-linear effects of inbreeding. Thompson et al. (2000) found a decrease in milk yield of $35 \mathrm{~kg}$ per $1 \%$ increase in inbreeding between 0 and $7 \%$, compared to a $55 \mathrm{~kg}$ per $1 \%$ increase for higher levels of inbreeding in Holstein cows, this again indicating a non-linear effect on milk yield.

The most common method used to estimate effects of inbreeding is linear regression of production on inbreeding coefficient (e.g., Falconer and MacKay, 1996). The regression coefficient is the expected change in the trait of interest per $1 \%$ increase in inbreeding and is a measure of inbreeding effects. Few studies have used non-linear regression of production on inbreeding coefficient. McParland et al. (2007), when comparing models with higher order polynomials and a classification model, reported significant quadratic effects when considering inbreeding as either a continuous variable 
or a fixed factor. Nevertheless, they urged caution in interpreting nonlinear results, due to large-standard errors in estimates at higher levels of inbreeding. Croquet et al. (2007) compared the fit of linear $\left(y=\beta_{0}+\beta_{1} X+e\right)$, quadratic $\left(y=\beta_{0}\right.$ $\left.+\beta_{1} X+\beta_{2} X^{2}+e\right)$ and cubic $\left(y=\beta_{0}+\beta_{1} X+\beta_{2} X^{2}+\beta_{3} X^{3}+e\right)$ regression models for estimating the effects of inbreeding based on milk yield. The coefficients in all the three methods were significantly different from zero. The largest t-value was for the simple linear regression coefficient. They proposed using the linear regression model for estimating the effects of inbreeding, especially for animals less than $10 \%$ inbred. Gulisija et al. (2007) used a non-parametric approach to estimate inbreeding effects on production in Jerseys. They reported that, on including inbreeding as a linear covariate, the fit of the model at low levels of inbreeding improved $(<$ $7 \%$ ). The lack of fit was detected in the linear regression model at higher levels of inbreeding (>10\%), for which a third-order regression model seemed to adequately fit the data. Another method which could be used is cubic-spline interpolation to estimate the non-linear effects of inbreeding on milk yield. The rationale is that this method, besides providing better coverage of data points (Harrell Jr, 2001), may result in more accurate estimates of the effects of inbreeding on milk yield.

The objective of this study was to compare cubicspline interpolation to estimate effects of inbreeding on milk yield with traditional linear regression and fixed factor models.

\section{Materials and Methods}

\section{Data and edits}

Records of actual milk yield adjusted to 305-d in lactation (2X) for first-calf Holstein heifers, freshening between 2002 and 2006, were used. The records were provided by the Dairy Herd Information Association (DHIANorth Carolina). Only records of registered Holstein heifers were included in the analysis. Animals with less than five recorded test-day milk yields were deleted from the data. Records of annual milk yield included in the data set, consisted of milk records within four times the standard deviation from the average (approximately 9,100 kg). As standard deviation was approximately $1,660 \mathrm{~kg}$, rounding up the confidence range to the nearest $100 \mathrm{~kg}$ resulted in some yields that were less than $2,400 \mathrm{~kg}$, and others more than $15,800 \mathrm{~kg}$. Through being considered as outliers, these were omitted from the data set. Contemporary groups were herd-year-seasons (HYS) of freshening, with season 1 defined as October 1 through March 31, and season 2 defined as April 1 through September 30. Records of HYS with less than 10 freshening heifers were deleted. Pedigree information was provided by the Holstein Association, USA. The pedigree file used was extended backwards, so that at least one of the paternal and maternal grandsires or granddams was known for all the heifers included in the analysis. Re- cords of animals that did not fit the minimum requirements were omitted. Nonetheless, these animals were included in the pedigree file, which finally consisted of 541,249 animals. Individual inbreeding coefficients $(\% \mathrm{~F})$ were provided by AIPL, as calculated by Wiggans et al. (1995). After edits, the analysis included records of 123,639 heifers in 5,839 HYSs, with individual inbreeding coefficients ranging from 0 to $40 \%$. Few animals (approximately $0.1 \%$ ) had levels of inbreeding greater than $18.75 \%$. The distribution of inbreeding coefficients was similar to that found in previous studies (e.g., Hudson and Van Vleck, 1984a,b; Miglior et al., 1992). Figure 1 presents average unadjusted milk yields with values within one standard deviation) by inbreeding class. Fluctuation of averages for inbreeding coefficients greater than $12 \%$ may be due to the small number of animals in those categories. In fact, milk yield records of animals with inbreeding greater than $12 \%$ constituted less than $1 \%$ of the available data, with several of these inbreeding levels ( 8 out of 19) having less than 10 observations each.

\section{Statistical models}

All the models included a random animal genetic effect with HYS treated as a fixed factor. The ten models compared were grouped as follows:

\section{Fixed factor models}

The first model was a saturated fixed-factor model, where the inbreeding coefficients (F) were included as levels of a fixed factor. Inbreeding coefficients rounded to the nearest integer were considered as unique levels. Thus, 32 different inbreeding levels were formed ( $0 \%$ to $40 \%$, with levels 22, 24 and 33 through 39 missing).

The second fixed factor model consisted of grouping $\mathrm{F}$ into seven classes as shown in Table 1. This classification has been used in several studies (e.g., Hudson and Van Vleck, 1984a,b; Miglior et al., 1992; McParland et al., 2007).The general form of the fixed factor models is:

$$
y_{i j k l}=\mu+F_{i}+H Y S_{j}+\text { Animal }_{k}+e_{i j k l}
$$

with $y_{i j k l}$, milk yield of Animal $k$ in HYS $j$ with an inbreeding coefficient $\mathrm{F}$ falling within inbreeding level $i$; $\mu$, a con-

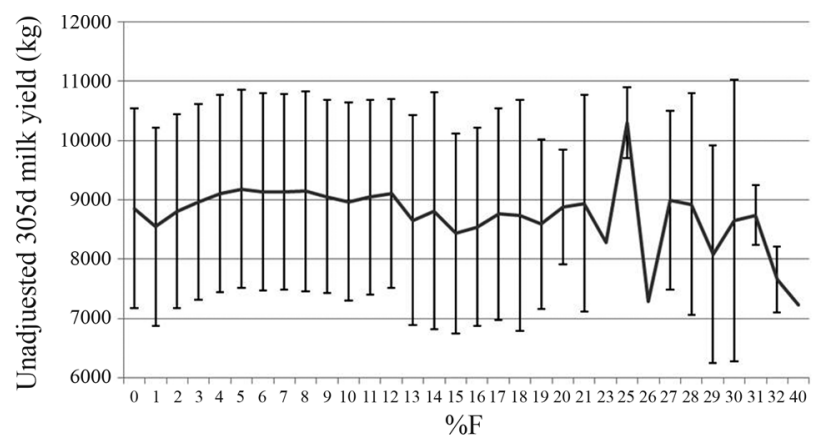

Figure 1 - Unadjusted average 305 d milk yield $(\mathrm{kg})$ with values within 1 standard deviation by inbreeding coefficient $(\% \mathrm{~F})$. 
Table 1 - Classification of inbreeding into seven levels, and the number of cows per category.

\begin{tabular}{lc}
\hline Inbreeding levels & Numberof cows \\
\hline $0 \%$ & 794 \\
$0-3.125 \%$ & 16308 \\
$3.125-6.250 \%$ & 81805 \\
$6.250-12.500 \%$ & 23832 \\
$12.500-18.750 \%$ & 780 \\
$18.750-25.000 \%$ & 36 \\
$>25.000 \%$ & 84 \\
\hline
\end{tabular}

stant; $F_{I}$, the effect of the $i^{\text {th }}$ level of inbreeding for $i=1, \ldots$, 32 with the saturated fixed factor model, and $i=1, \ldots, 7$ with the fixed factor model; $H Y S_{j}$, the effect of the $j^{\text {th }}$ HYS contemporary group treated as a fixed factor; Animal $_{k}$, a random additive genetic value of the $k^{\text {th }}$ animal with $\sigma_{a}^{2}=0.5 \sigma_{e}^{2}$, and $e_{i j k l}$, a random error effect normally and independently distributed with a mean of zero and a variance, $\sigma_{e}^{2}$.

\section{Regression models}

Inbreeding coefficients were included as covariates with linear, linear and quadratic or linear, quadratic and cubic effects, resulting in three models.

The linear regression model was:

$$
y_{i j k}=\beta_{0}+\beta_{1} F+H Y S_{i}+\text { Animal }_{j}+e_{i j k}
$$

with $y_{i j k}$, milk yield of Animal $j$ in HYS $i$ with an inbreeding coefficient $F ; \beta_{0}$, intercept; $\beta_{1}$, regression coefficient for the linear effect of inbreeding; $F$, inbreeding coefficient of animal $j$; HYS, Animal and $e$ effects as defined earlier.

The linear and quadratic regression model was:

$$
y_{i j k}=\beta_{0}+\beta_{1} F+\beta_{2} F^{2}+H Y S_{i}+\text { Animal }_{j}+e_{i j k}
$$

with $y_{i j k}$, milk yield of Animal $j$ in HYS $i$ with an inbreeding coefficient $\mathrm{F} ; \beta_{0}$, intercept; $\beta_{1}$, coefficient of the linear effect of inbreeding; $F$, inbreeding coefficient of animal $j ; \beta_{2}$, coefficient for the quadratic effect of inbreeding; and HYS, Animal and $e$ effects as defined earlier.

The cubic regression model was:

$$
y_{i j k}=\beta_{0}+\beta_{1} F+\beta_{2} F^{2}+\beta_{3} F^{3}+H Y S_{i}+\text { Animal }_{j}+e_{i j k}
$$

with $y_{i j k}$, milk yield of Animal $j$ in HYS $i$ with an inbreeding coefficient $\mathrm{F} ; \beta_{0}$, intercept; $\beta_{1}$, coefficient of the linear effect of inbreeding; $F$, inbreeding coefficient of animal $k ; \beta_{2}$, coefficient for the quadratic effect of inbreeding; $\beta_{3}$, coefficient for the cubic effect of inbreeding; and HYS, Animal and $e$ effects as defined earlier.

\section{Cubic-spline models}

The spline models had three to seven knots $\left(t_{3} \ldots t_{7}\right)$ for F. Choice of knots was based on consideration of the different possible inbreeding coefficients that could be attained from mating of directly related animals such as full-sibs, half-sibs, parent- progeny and grandparent-grandprogeny matings. Some knots were also chosen to divide the inbreeding levels into equally spaced groups. For the cubic-spline model with seven knots, the knots were chosen to match the seven levels of the fixed factor model. The knot positions are presented in Table 2. This approach resulted in five different models, the most complete being:

$$
y_{i j k}=\beta_{0}+\beta_{1} F+\beta_{2} F^{2}+\beta_{3} F^{3}+H Y S_{i}+\text { Animal }_{j}+e_{i j k}
$$

with $y_{i j k}$, milk yield of Animal $j$ in HYS $i$ with an inbreeding coefficient $F ; \beta_{0}$, intercept; $\beta_{1}$, coefficient for the linear effect of inbreeding; $F$, inbreeding coefficient of animal $j$; $\beta_{1}, \ldots, \beta_{6}$, the Z-spline coefficients of the cubic-spline interpolation for $\mathrm{F} ; F_{2}, \ldots, F_{6}$, the knot functions; and HYS, Animal and $e$ effects as previously described.

Depending on the number of knots in the model, some terms were removed.

Other models could have also been used; for example, higher order linear polynomials, such as quartic and quintic. Inclusion of other fixed and /or random effects might improve the fit of the model. The focus of the project was to compare cubic-spline interpolation with traditionally used models. Thus, the models used were kept as similar as possible to the traditional models. Furthermore, as discussed later, comparisons required fixed genetic variance. Animal genetic variance was chosen to match heritability estimates from other studies (e.g., Swalve and VanVleck, 1987).

All analyses were conducted using ASREML 2.0 (Gilmour et al., 2006).

\section{Model comparisons}

Models were compared using Akaike's Information Criterion (AIC; Akaike, 1974), one of the several model selection methods based on the principle of parsimony. Other methods based on the same principle could have been used but the AIC is one of the easiest methods to compute and does not require extensive computation. Model selection could have been also conducted in the context of null hypothesis testing, however this approach has relatively poor performance given its dependence on the significance level specified to test if effects should be included or omitted

Table 2 - Position of knots at inbreeding levels $(\% \mathrm{~F})$ for the five cubic-spline models.

\begin{tabular}{ll}
\hline Number of knots & Position of knots \\
\hline 3 & $0,12.500,25.000$ \\
4 & $0,6.250,12.500,25.000$ \\
5 & $0,3.125,6.250,12.500,25.000$ \\
6 & $0,3.125,6.250,12.50,18.750,25.000$ \\
7 & $0,3.125,6.250,12.50,18.750,25.000,37.500$ \\
\hline
\end{tabular}


from a model ( $\alpha=0.05$ or 0.01 or 0.15$)$. The null hypothesis testing approach also has a relatively poor performance when testing non-nested models (Burnham and Anderson, 2002). The AIC is an approximate measure of the Kullback-Leibler information of a model based on the estimate of log-likelihood and number of parameters estimated: $A I C=2 p-2 l$, where $p$ is the number of parameters estimated, and $l$ is the log-likelihood of the model used. When the ratio of the number of observations, $n$, to the number of parameters estimated, $p$, is less than 40 , it is recommended that corrected AIC be used: $\mathrm{AICc}=\mathrm{AIC}+[2 p(p$ $+1) /(n-p-1)]$ (Burnham and Anderson, 2002). As the number of observations becomes large, AICc converges to AIC. The idea behind AIC is that the difference between two competing models, A and B, can be detected by differences in estimates of residual error variances from the two models. As, by increasing the number of parameters in a model, the goodness of fit improves, i.e., there is a reduction in the residual sum of squares and an increase in log-likelihood in nested models, a growing penalty function, equal to twice the number of parameters included, is deducted to discourage "overfitting". One disadvantage of overfitting is that, given enough parameters, an irrational model may fit the data perfectly, even if it does include nonsensical parameters. Models were ranked according to their AICc because the ratio of the number of observations to the number of parameters estimated, was always less than 40. The model with the smallest AICc was considered the best. The significance of differences between models was based on their AICc values, as described by Burnham and Anderson (2002) for comparison of models. In general, a small difference in AICc between two competing models (less than 2) indicates that neither of the two is adequate. A moderate difference (4 to 7) indicates that the model with the higher AICc does not fit the data as well as the one with the lower AICc rank. A large difference (greater than 10) indicates that the model with the highest AICc is inadequate compared to that with the lowest. The log-likelihood used in the AIC, and subsequently the AICc, is based on maximizing likelihood (ML). The software used in the analysis however maximized the restricted maximum likelihood (REML). Comparison of the different models therefore required manipulation of the likelihood estimates from REML to be converted into ML estimates. Therefore, the animal genetic variance was fixed for all models to facilitate estimating random residual variance. Heritability of milk yield at first lactation was assumed to be 0.33 (Swalve and Van Vleck, 1987; Miglior et al., 1992). The additive genetic component of variance was fixed at half residual-error variance to facilitate comparing models. With genetic variance as a fixed function of residual-error variance, only the residual-variance component needed to be estimated. The next step was to convert the REML estimate of residual-error variance into the ML estimate for computing likelihood and AIC. The method of transformation from REML to ML estimates is as follows:

The REML estimate of residual-error variance, $\hat{\sigma}_{e_{\text {PEVI }}}^{2}$, is equal to the sum of squares of the residuals (SSR), divided by the degrees of freedom for error $\left(d f_{e}\right)$, whereas the ML estimate of residual-error variance, $\hat{\sigma}_{M L}^{2}$ is equal to SSR divided by the total number of observations $(n)$. This leads to the following adjustment: $\hat{\sigma}_{e_{R E M L}}^{2}=S \hat{S} R / d f_{e}$ and $\hat{\sigma}_{e_{M L}}^{2}=S \hat{S} R / n$, then $S \hat{S} R=d f_{e} \times \hat{\sigma}_{e_{R E M L}}^{2}=n \times \hat{\sigma}_{e_{M L}}^{2}$ so that $\sigma_{e_{M L}}^{2}=\left(d f_{e} / n\right) \times \sigma_{e_{R E M L}}^{2}$.

Milk yield was assumed to be approximately normally distributed, $y \sim N\left(X \beta, V \sigma_{e}{ }^{2}\right)$, so that the likelihood function given $y$ is:

$$
\begin{aligned}
& L\left(\beta, \sigma_{e}^{2} \mid y\right)=\left(\frac{1}{\left(\sqrt{2 \pi \sigma_{e}^{2}}\right)}\right)^{n}|V|^{-n / 2} \times \\
& \exp \left[-\frac{1}{2}(y-X \beta)^{\prime}\left(V \sigma_{e}^{2}\right)^{-1}(y-X \beta)\right]
\end{aligned}
$$

where $y$ is the $n \times 1$ vector of observations; $n$ is total number of observations; $V$ is a matrix of constants, since the animal genetic variance was fixed at one-half residual-error variance; $\sigma_{e}{ }^{2}$ is the residual-error variance; and $\sigma_{e}^{2}$ is the product of the design matrix, $X$, and the vector of fixed effects, $\beta$.

Thus, the log-likelihood function is:

$$
\begin{aligned}
& l\left(\beta, \sigma_{e}^{2} \mid y\right)=-\frac{n}{2} \ln (2 \pi)-\frac{n}{2} \ln \left(\sigma_{e}^{2}\right)-\frac{n}{2} \ln |V|- \\
& \frac{1}{2}\left[\frac{(y-X \beta)^{\prime} V^{-1}(y-X \beta)}{\sigma_{e}^{2}}\right]
\end{aligned}
$$

To find the estimate of $\sigma_{e}{ }^{2}$ that maximizes likelihood, the first derivative with respect to $\sigma_{e}^{2}$ :

$$
\frac{\delta l\left(\beta, \sigma_{e}^{2} \mid y\right)}{\delta \sigma_{e}^{2}}=0-\frac{n}{2 \sigma_{e}^{2}}-0+\frac{1}{2}\left(\frac{(y-X \beta)^{\prime} V^{-1}(y-X \beta)}{\sigma_{e}^{4}}\right)
$$

is equated to zero. This permits estimating $\sigma_{e}{ }^{2}$ from

$$
\frac{n}{2 \hat{\sigma}_{e}^{2}}=\frac{1}{2}\left(\frac{(y-X \beta)^{\prime} V^{-1}(y-X \beta)}{\sigma_{e}^{4}}\right)
$$

and

$$
\frac{\sigma_{e}^{4} n}{2 \sigma_{e}^{2}}=\frac{1}{2}\left[(y-X \hat{\beta})^{\prime} V^{-1}(y-X \hat{\beta})\right]
$$

so that

$$
\sigma_{e_{M L}}^{2}=\frac{(y-X \hat{\beta})^{\prime} V^{-1}(y-X \hat{\beta})}{n}
$$


The log-likelihood is therefore:

$$
\begin{aligned}
& l\left(\hat{\beta}, \hat{\sigma}_{e_{M L}}^{2} \mid y\right)=-\frac{n}{2} \ln (2 \pi)-\frac{n}{2} \ln \left(\hat{\sigma}_{e_{M L}}^{2}\right)-\frac{1}{2} \ln |V|- \\
& \frac{1}{2}\left[\frac{(y-X \hat{\beta})^{\prime} V^{-1}(y-X \hat{\beta})}{\hat{\sigma}_{e_{M L}}^{2}}\right]=-\frac{n}{2} \ln (2 \pi)- \\
& \frac{n}{2} \ln \left(\hat{\sigma}_{e_{M L}}^{2}\right)-\frac{1}{2} \ln |V|-\frac{1}{2}\left[\frac{n \hat{\sigma}_{e_{M L}}^{2}}{\hat{\sigma}_{e_{M L}}^{2}}\right]
\end{aligned}
$$

so that

$$
l\left(\hat{\beta}, \hat{\sigma}_{e_{M L}}^{2} \mid y\right)=-\frac{n}{2} \ln (2 \pi)-\frac{n}{2} \ln \left(\hat{\sigma}_{e_{M L}}^{2}\right)-\frac{1}{2} \ln |V|-\frac{n}{2}
$$

Because $n, 2 \pi$ and $V$ are constants,

$$
l\left(\hat{\beta}, \hat{\sigma}_{e_{M L}}^{2} \mid y\right)=\mathrm{constant}-\frac{n}{2} \ln \left(\hat{\sigma}_{e_{M L}}^{2}\right) .
$$

The constant is the same for all models because $n, 2 \pi$ and $V$ are the same for all models.

\section{Comparison of models for inbreeding effects}

The analyses were re-run without restrictions on the animal genetic variance to obtain, not only estimates of variance components and predicted animal breeding values for traditional linear regression and fixed factor models, but also the best cubic-spline model for comparing estimates of the effects of inbreeding on milk yield and of variance components and heritability. For each model, the predicted breeding values (EBV) for sires of daughters with records were compared to detect changes in sire-ranking due to the model used. The pedigree file contained 9,618 sires of which 1,409 had daughters with records. The proc reg procedure in $\mathrm{SAS}^{\odot} 9.2$ was used to regress EBVs of these sires from the cubic-spline model, on the corresponding EBV from the fixed factor and linear regression models. The calculated correlations among EBV of sires of daughters with records from all three methods were examined using the proc corr procedure in $\mathrm{SAS}^{\odot}$ 9.2. Ranking of the top 100 sires for the three models were also compared. Finally, the estimated milk yields by inbreeding level using the best model were regressed on estimated milk yields by inbreeding level from the linear regression and fixed factor models using the proc reg procedure in $\mathrm{SAS}^{\odot}$ 9.2.

\section{Results and Discussion}

Estimates of residual-error variance, log-likelihood, and AICc for each model, as well as differences in AICc from the model with the lowest AICc (assumed best), are given in Table 3. Based on AICc, the best model was cubic-spline interpolation of inbreeding with seven knots. The model with the highest AICc was the saturated fixed factor model (a difference of +50.447 from the best model). The fixed factor model and simple linear regression model, i.e. the traditional methods of analysis, ranked second and third to last, respectively. The cubic-spline model with 4 knots had 0.48 higher AICc than the model with seven knots indicating that the two models are nearly equivalent. The cubic-spline models with five and six knots as well as the cubic regression model had AICc differences from the best model ranging between 2 and 6 reflecting that these models do not fit the data as well as the best model. The remaining models (i.e. linear regression, linear and quadratic regression, cubic-spline with three knots, fixed factor and saturated models) all had differences of AICc larger than 10 units compared with the best model indicating a poor fit to the data compared to the cubic-spline with seven knots.

The counter-intuitive result of the cubic-spline models with five and six knots, which performed worse than that with four, is explained by differences in log-likelihoods and the number of parameters between the models. Compared to the model with four knots, one additional parameter was estimated in the model with five knots, and two in that with 6 . Even though this difference was small, it lead to AICc ranking differing from what was to be expected.

Table 3 - Estimates of residual variance, logarithm of the likelihood and AICc for each model, with differences in AICc from the cubic-spline model with seven knots.

\begin{tabular}{lcccc}
\hline Model & $\hat{\sigma}_{e}^{2}\left(\mathrm{~kg}^{2}\right)(\mathrm{x} 10,000)$ & $\operatorname{LogL}(-865,000)$ & AICc $(1,742,500)$ & Difference in AICc \\
\hline Cubic-spline with 7 knots & 125.515 & -122.913 & 15.995 & 0 \\
Cubic-spline with 4 knots & 125.519 & -126.458 & 16.473 & 0.479 \\
Cubic-spline with 5 knots & 125.520 & -126.425 & 18.612 & 2.618 \\
Cubic-spline with 6 knots & 125.521 & -126.393 & 20.751 & 4.756 \\
Linear, quadratic and cubic regression & 125.524 & -128.920 & 21.398 & 5.404 \\
Linear and quadratic regression & 125.532 & -133.385 & 28.124 & 12.130 \\
Cubic-spline with 3 knots & 125.534 & -134.370 & 30.094 & 14.100 \\
Linear regression & 125.539 & -137.357 & 33.865 & 17.870 \\
Fixed factor 7 & 125.546 & -138.180 & 50.934 & 34.940 \\
Saturated (fixed factor 32 levels) & 125.509 & -118.383 & 66.441 & 50.447 \\
\hline
\end{tabular}


Estimates of the effects of inbreeding on milk yield for a cubic-spline model with seven knots are represented in Figure 2. Table 4 contains t-values to test for significance of reduction in $305 \mathrm{~d}$ milk yield between each inbreeding level and no inbreeding. Significant differences at the 0.05 rejection level were at $\mid t$-value $\mid>1.96$. These results showed no significant decrease in milk production up to an inbreeding level of $8 \%$. Milk yields significantly decreased at inbreeding levels of $9 \%$ to $21 \%$. No significant differences in milk yields were found for inbreeding levels of $23 \%$ to $27 \%$. Significant differences in milk yields were detected for inbreeding levels of $28 \%$ and beyond.

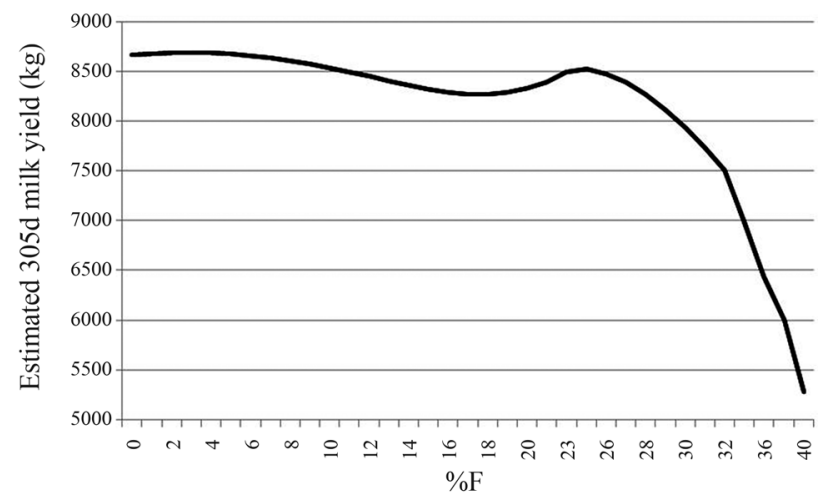

Figure 2 - Estimated $305 \mathrm{~d}$ milk yields $(\mathrm{kg})$ by inbreeding level $(\% \mathrm{~F})$ from the cubic-spline model adjusted for herd-year-season effects.
The linear regression model indicated a decrease of $21.49 \mathrm{~kg}$ of milk per $1 \%$ increase in inbreeding, in agreement with estimates in the literature for Holstein cattle, which ranged from 9.84 to $26.00 \mathrm{~kg}$ (Thompson et al., 2000). Estimates of losses due to inbreeding using the fixed factor model are represented in Table 5. These estimates generally agree with previous studies in which five inbreeding classes were used (Hudson and Van Vleck, 1984a,b; Miglior et al., 1992; Thompson et al., 2000).

Estimates of variance components and heritability for the cubic-spline model with seven knots, for the linear regression model and for the fixed factor model with seven levels are shown in Table 6 . The three models resulted in similar estimates of variance components and heritability

Table 5 - Estimated annual milk yield loss $(\mathrm{kg})$ at different inbreeding levels for the fixed factor model with seven levels.

\begin{tabular}{lc}
\hline Inbreeding levels & Milk yield loss $(\mathrm{kg})$ \\
\hline $0^{+}-3.125 \%$ & $5.92 \pm 18.85$ \\
$3.125^{+}-6.25 \%$ & $78.64 \pm 21.15$ \\
$6.25^{+}-12.5 \%$ & $386.10 \pm 52.45$ \\
$12.5^{+}-18.75 \%$ & $366.17 \pm 208.70$ \\
$18.75^{+}-25 \%$ & $479.75 \pm 148.62$ \\
$>25 \%$ & $2863.37 \pm 1291.89$ \\
\hline
\end{tabular}

Table 4 - $\mathrm{t}$ statistics for testing significance in the reduction of $305 \mathrm{~d}$ milk yield $(\mathrm{kg})$ between different inbreeding levels and $0 \%$ inbreeding from the cubic-spline interpolation model with seven knots.

\begin{tabular}{|c|c|c|c|c|c|}
\hline$\% \mathrm{~F}$ & $305 \mathrm{~d}$ milk yield reduction per cow $(\mathrm{kg})$ & t-value & $\% \mathrm{~F}$ & $305 \mathrm{~d}$ milk yield reduction per cow $(\mathrm{kg})$ & t-value \\
\hline 0.00 & 0.00 & 0.00 & 20.00 & -332.00 & $-3.46^{*}$ \\
\hline 1.00 & 13.00 & 0.75 & 21.00 & -276.00 & $-2.47^{*}$ \\
\hline 2.00 & 22.00 & 0.72 & 23.00 & -170.00 & -1.12 \\
\hline 3.00 & 25.00 & 0.64 & 25.00 & -146.00 & -0.84 \\
\hline 4.00 & 21.00 & 0.49 & 26.00 & -191.00 & -1.11 \\
\hline 5.00 & 10.00 & 0.20 & 27.00 & -275.00 & -1.68 \\
\hline 6.00 & -9.00 & -0.27 & 28.00 & -394.00 & $-2.56^{*}$ \\
\hline 7.00 & -32.00 & -0.91 & 29.00 & -546.00 & $-3.50^{*}$ \\
\hline 8.00 & -61.00 & -1.64 & 30.00 & -727.00 & $-4.04^{*}$ \\
\hline 9.00 & -95.00 & $-2.38^{*}$ & 31.00 & -933.00 & $-4.10^{*}$ \\
\hline 10.00 & -132.00 & $-3.05^{*}$ & 32.00 & -1162.00 & $-3.95^{*}$ \\
\hline 11.00 & -172.00 & $-3.70^{*}$ & 34.00 & -1673.00 & $-3.61^{*}$ \\
\hline 12.00 & -216.00 & $-4.40^{*}$ & 36.00 & -2231.00 & $-3.38^{*}$ \\
\hline 13.00 & -262.00 & $-5.18^{*}$ & 40.00 & -3390.00 & $-3.13^{*}$ \\
\hline 14.00 & -308.00 & $-5.85^{*}$ & & & \\
\hline 15.00 & -349.00 & $-6.12^{*}$ & & & \\
\hline 16.00 & -381.00 & $-5.98^{*}$ & & & \\
\hline 17.00 & -399.00 & $-5.60^{*}$ & & & \\
\hline 18.00 & -399.00 & $-5.08^{*}$ & & & \\
\hline 19.00 & -377.00 & $-4.39^{*}$ & & & \\
\hline
\end{tabular}

*Significant reduction in milk yield from the estimated milk yield at $0 \%$ inbreeding $(\mathrm{p}<0.05)$. 
Table 6 - Estimates of variance components $(\mathrm{kg} / 100)^{2}$ and heritability for the three models.

\begin{tabular}{lccc}
\hline Model & $\sigma_{a}^{2}$ & $\sigma_{p}^{2}$ & Heritability \\
\hline Linear regression & $59.50 \pm 2.24$ & $187.40 \pm 0.98$ & $0.31 \pm 0.01$ \\
Fixed factor with seven levels & $58.90 \pm 2.23$ & $187.20 \pm 0.98$ & $0.31 \pm 0.01$ \\
Cubic-spline with seven knots & $58.97 \pm 2.24$ & $187.20 \pm 0.98$ & $0.31 \pm 0.01$ \\
\hline
\end{tabular}

of approximately $0.31(\mathrm{SD}=0.01)$ which agrees with estimates of heritability reported in the literature of 0.33 (e.g., Swalve and Van Vleck, 1987).

Regression of EBV from the cubic-spline model on EBV from the linear regression model for the 1,409 sires having daughters with records, resulted in $\mathrm{R}^{2}$ of 0.99 , a correlation of 0.99 and a regression coefficient of 0.97 . Regression of EBV from the cubic-spline model on EBV from the fixed factor model had a $\mathrm{R}^{2}$ of 0.99 , a correlation of 0.99 and a regression coefficient of 0.99 . The regression coefficient from regression of EBV from the cubic-spline model on the EBV from the linear regression model, was significantly different from $1.00(\mathrm{p}<0.0001)$. The regression coefficient of the regression of EBV from the cubic-spline model on the EBV from the fixed factor model, was not significantly different from 1.00 $(\mathrm{p}=0.31)$. Correlations between EBV from the cubicspline model and EBV from the linear regression and fixed factor models were both significantly different from $1.00(\mathrm{p}<0.0001)$, when the Fisher $(\mathrm{rho} 0=1.00)$ option was specified in the CORR procedure in SAS ${ }^{\odot}$. Thus, estimates of breeding values could possibly be different for the three models. Nonetheless, ranking of the top 100 sires by each method revealed that only the rank of one sire changed between the linear regression model and the two other models indicating that selection for breeding value was minimally affected by the model used.

The regression of milk yield estimates by inbreeding level from the cubic-spline model on milk yield estimates by inbreeding level from the linear regression model had a $\mathrm{R}^{2}$ of 0.99 , a correlation of 0.79 and a regression coefficient of 0.97 that was significantly different from $1.00(\mathrm{p}=0.02)$. The regression of estimates by inbreeding level from the cubic-spline model on estimates by inbreeding level from the fixed factor model had a $\mathrm{R}^{2}$ of 0.99 , a correlation of 0.88 and a regression coefficient of 0.98 that was not significantly different from $1.00(\mathrm{p}=0.13)$.

Consequently, the linear regression model is apparently not the best for estimating the effects of inbreeding on milk yield. Despite its lower AICc ranking, the fixed factor model appears to be a simpler and more effective alternative to the more complex cubic-spline. Estimates of the effects of inbreeding on milk production were similar for the cubic-spline model and the fixed factor model. This may be due to the positions of the knots in the cubic-spline model which coincide, for the most part, with the inbreeding levels for the fixed factor model.
A substantial reduction in profit is reflected from losses derived from the detrimental effects of inbreeding. At an inbreeding level of $9 \%$, the estimated loss in milk yield would be $95 \mathrm{~kg}$, thereby reflecting a potential loss of US\$ 26.18 per lactation at the average milk-price of US\$ 12.5 per hundred weight. The average inbreeding in the U.S. Holstein population was $5.50 \%$ for the year 2009 , and is on the increase, as reported by the Animal Improvement Program Laboratory.

In this study, knots were positioned at inbreeding levels reflecting the possible mating of directly related animals (within a family line). Therefore, given the similarity in estimates of effects of inbreeding on milk yield, the fixed factor model, despite poorer AICc, presents a simple and effective alternative to using the complex cubic-spline.

If adjustment of milk yields for inbreeding is to be based on estimates using a cubic-spline model, it would be advantageous to develop an algorithm, useful in detecting the positions of knots providing the best fit to the data. Their positioning would first need to be defined in one or several reference data sets, and then validated in others. $\mathrm{Cu}-$ bic-spline coefficients would require periodical re-validation.

\section{Acknowledgments}

This manuscript is a contribution of the University of Nebraska Agricultural Research Division, supported in part by funds provided through the Hatch Act, USDA.

The authors would like to thank the Holstein Association U.S.A for providing the pedigree file, as well as the Animal Improvement Program Laboratory of ARS-USDA for providing estimates of inbreeding coefficients on the cows used in this study. The authors also wish to express their gratitude to Dr. Stephen Kachman for guidance and assistance in model comparison.

\section{References}

Akaike H (1974) A new look at the statistical model identification. IEEE Transact Autom Control 19:716-723.

Burnham KP and Anderson DR (2002) Model Selection And Multimodel Inference, 2nd edition. Springer, New York, 353 pp.

Croquet C, Mayeres P, Gillon A, Hammami H, Soyeurt H, Vanderick S and Gengler N (2007) Linear and curvilinear effects of inbreeding on production traits for Walloon Holstein cows. J Dairy Sci 90:465-471. 
Falconer DS and MacKay TFC (1996) Introduction to Quantitative Genetics. 4th edition. Addison Wesley Longman, Harlow, $438 \mathrm{pp}$.

Gilmour AR, Gogel BJ, Cullis BR and Thompson R (2006) ASReml User Guide Release 2.0 VSN International Ltd, Hemel Hempstead.

Gulisija D, Gianola D and Weigel KA (2007) Nonparametric analysis of the impact of inbreeding on production in Jersey cows. J Dairy Sci 90:493-500.

Harrell Jr FE (2001) Regression Modeling Strategies. Springer, NewYork, 568 pp.

Hudson GFS and Van Vleck LD (1984a) Inbreeding of artificially bred dairy cattle in the Northern United States. J Dairy Sci 67:161-170.

Hudson GFS and Van Vleck LD (1984b) Effects of inbreeding on milk and fat production, stayability, and calving interval of registered Ayrshire cattle in the Northeastern United States. J Dairy Sci 67:171-179.

Kearney JF, Wall E, Villaneuva B and Coffey MP (2004) Inbreeding trends and application of optimized selection in the UK Holstein population. J Dairy Sci 87:3503-3509.

McParland S, Kearney F, Rath M and Berry DP (2007) Inbreeding effects on milk production, calving performance, fertility and conformation in Irish Holstein-Friesians. J Dairy Sci 90:4411-4419.
Miglior F, Szkotnicki B and Burnside EB (1992) Analysis of levels of inbreeding and inbreeding depression in Jersey cattle. J Dairy Sci 75:1112-1118.

Swalve H and Van Vleck LD (1987) Estimation of genetic (Co) variances for milk yield in first three lactations using an animal model and restricted maximum likelihood. J Dairy Sci 70:842-849.

Thompson JR, Everett RW and Hammerschmidt NL (2000) Effects of inbreeding on production and survival in Holsteins. $\mathrm{J}$ Dairy Sci 83:1856-1864.

Weigel KA (2001) Controlling inbreeding in modern breeding programs. J Dairy Sci 84:E177-E184.

Wiggans GR, VanRaden PM and Zuurbier J (1995) Calculation and use of inbreeding coefficients for genetic evaluation of United States dairy cattle. J Dairy Sci 78:1584-1590.

\section{Internet Resources}

Animal Improvement Program Laboratory (ARS-AIPL) August 2009 Inbreeding information http://aipl.arsusda.gov/eval/ summary/inbrd.cfm (September 14, 2009).

Associate Editor: Alexandre Rodrigues Caetano

License information: This is an open-access article distributed under the terms of the Creative Commons Attribution License, which permits unrestricted use, distribution, and reproduction in any medium, provided the original work is properly cited. 